\title{
Isovector pairing in odd-A proton-rich nuclei
}

\author{
Jonathan Engel \\ Dept. of Physics and Astronomy, CB3255, University of North Carolina, Chapel Hill, NC 27599 \\ Karlheinz Langanke \\ Inst. for Phys. and Astr. and Cen. for Theor. Astrophys., Univ. of Aarhus, DK-8000 Aarhus C, Denmark \\ Petr Vogel \\ Physics Department, California Institute of Technology, Pasadena, California 91125
}

August 8, 2018

\begin{abstract}
A simple model based on the group $\mathrm{SO}(5)$ suggests that both the likeparticle and neutron-proton components of isovector pairing correlations in odd- $A$ nuclei are Pauli blocked. The same effect emerges from Monte Carlo Shell-model calculations of proton-rich nuclei in the full $f p$ shell. There are small differences between the two models in their representation of the effects of an odd nucleon on the competition between like-particle and neutron-proton pairing, but they can be understood and reduced by using a two-level version of the $\mathrm{SO}(5)$ model. On the other hand, in odd-odd nuclei with $N \neq Z, \mathrm{SO}(5)$ disagrees more severely with the shell model because it incorrectly predicts ground-state isospins. The shell model calculations for any $f p$-shell nuclei can be extended to finite temperature, where they show a decrease in blocking.
\end{abstract}


Proton-rich nuclei play an important role in explosive nucleosynthesis and are increasingly accessible to experiment. For this reason the subject of neutron-proton pairing has experienced a revival. Even- $A$ nuclei have received most of the attention so far, but odd- $A$ nuclei, in which pairing is affected by the odd nucleon, are equally important. In a previous paper[1] we analyzed the competition between neutron-proton $(n p)$ and like-particle ( $p p$ and $n n$ ) pairing in even-even $f p$-shell nuclei and in odd-odd $N=Z$ nuclei, arguing that a simple model based on the group $\mathrm{SO}(5)$ captured the essentials of full shell-model calculations, despite deformation, spin-orbit splitting, and other physics that the simple model omits. Here we turn to odd- $A$ nuclei, discussing the same competition when an extra nucleon is present and pairing correlations are blocked. We also touch on odd-odd nuclei, of which only those with $N=Z$ were treated in Ref. [1]. Though our focus is on ground states, we briefly discuss the pairing competition at finite temperature as well.

We begin with the predictions of the simple model, a full description of which is in Ref. [1]. Briefly, the isovector angular-momentum-zero $n n, p p$, and $n p$ pair creation operators, the corresponding annihilation operators, the three isospin generators, and the number operator form the algebra $\mathrm{SO}(5)$. The Hamiltonian consists of three equally weighted pairing terms and has the ground-state expectation value

$$
E=-\frac{G}{2}\left[n\left(\Omega-\frac{n-6}{4}\right)-\nu\left(\Omega-\frac{\nu-6}{4}\right)+t(t+1)-T(T+1)\right]
$$

where $G$ is a the pairing-force strength constant, $n$ is the number of nucleons, $\Omega$ is half the number of (degenerate) single-particle levels, $T$ is the isospin, and $\nu$ and $t$ are the the seniority and "reduced isospin", which take the values $\nu=0, t=0$ in even-A ground states and $\nu=1, t=1 / 2$ in odd-A ground states. When $\nu=0$ Eq. (1) becomes

$$
E=-\frac{G}{2}\left[n\left(\Omega-\frac{n-6}{4}\right)-T(T+1)\right] \text {. }
$$

When $\nu=1$ we have

$$
E=-\frac{G}{2}\left[n\left(\Omega-\frac{n-6}{4}\right)-\left(\Omega+\frac{1}{2}\right)-T(T+1)\right] .
$$

These equations imply a blocking in odd- $A$ nuclei very similar to what would be present without $n p$ pairing; in fact in the ordinary like-particle seniority model the pairing energies along any even- $Z$ isotope chain, in which there are no odd-odd nuclei, differ only by a small constant from Eqs. (2) and (3). This prediction is confirmed by shell-model Monte Carlo calculations, which we describe shortly. Since the $n p$ pairing replaces some of the like-particle pairing when an isospin-symmetric interaction is used, it would seem that both pairing modes are blocked in odd- $A$ nuclei, though $p p$ pairing is apparently almost unaffected by an odd neutron.

This can be seen explicitly by examining the competition among $n n, p p$, and $n p$ pairs, something we discussed in even- $A$ nuclei in Ref. [1]. Defining pair "number operators" as in that paper, (for a brief but exhaustive discussion of these operators see Ref. [2]), we use techniques described in Ref. [3] to obtain for odd- $A$ ground states:

$$
\left\langle\mathcal{N}_{p p}\right\rangle=\frac{\left(T_{c}+1\right)\left(\mathcal{N}-T_{c}\right)\left(1-\frac{\mathcal{N}-T_{c}-3}{2 \Omega}\right)}{2 T_{c}+3}
$$




$$
\begin{aligned}
\left\langle\mathcal{N}_{n p}\right\rangle & =\frac{\left(\mathcal{N}-T_{c}\right)\left(1-\frac{\mathcal{N}-T_{c}-2}{2 \Omega}\right)}{2 T_{c}+3} \\
\left\langle\mathcal{N}_{n n}\right\rangle & =\frac{\left(T_{c}+1\right)\left(\mathcal{N}+T_{c}\right)\left(1-\frac{\mathcal{N}+T_{c}+1}{2 \Omega}\right)+\left(T_{c}+\frac{\mathcal{N}}{\Omega}\right)}{2 T_{c}+3}
\end{aligned}
$$

if $\mathcal{N} \equiv(n-1) / 2$ (the total number of pairs) and $T_{c} \equiv T-1 / 2$ (the core isospin) are both even or both odd, and

$$
\begin{aligned}
\left\langle\mathcal{N}_{p p}\right\rangle & =\frac{T_{c}\left(\mathcal{N}-T_{c}\right)\left(1-\frac{\mathcal{N}-T_{c}-1}{2 \Omega}\right)}{2 T_{c}+1} \\
\left\langle\mathcal{N}_{n p}\right\rangle & =\frac{\left(\mathcal{N}-T_{c}+1\right)\left(1-\frac{\mathcal{N}-T_{c}-1}{2 \Omega}\right)}{2 T_{c}+1} \\
\left\langle\mathcal{N}_{n n}\right\rangle & =\frac{T_{c}\left(\mathcal{N}+T_{c}\right)\left(1-\frac{\mathcal{N}+T_{c}-3}{2 \Omega}\right)+\left(T_{c}-1\right)\left(1-\frac{\mathcal{N}+T_{c}}{\Omega}\right)}{2 T_{c}+1}
\end{aligned}
$$

if $\mathcal{N}$ and $T_{c} \equiv T+1 / 2$ are both even or both odd (the relation between $T_{c}$ and $T$ is different in the two sets of expressions). Combining these results with those for even- $A$ nuclei from Ref. [1], we plot on the left-hand side of Figure 1 the numbers of each kind of pair (scaled by 0.5 ; we discuss this factor shortly) as neutron number increases in the $\mathrm{Cr}$ isotopes. As the figure shows, $\left\langle\mathcal{N}_{n n}\right\rangle$ oscillates sharply while the other pair-numbers stagger less. The reason is that when $N$ is even as well as $Z$, $n n$ and $p p$ pairing (particularly the former for $N>Z$ ) are enhanced at the expense of $n p$ pairing. Adding a neutron to make $N$ odd blocks the $n n$ pairing, thereby reducing $\left\langle\mathcal{N}_{n n}\right\rangle$, but also blocks $n p$ pairing to a degree so that $\left\langle\mathcal{N}_{n p}\right\rangle$ cannot take advantage of the reduced coherence in the $n n$ condensate. A little surprisingly, perhaps, $\left\langle\mathcal{N}_{p p}\right\rangle$ is not able to take any advantage of the slight drop in $n p$ pairing; it doesn't increase until $N$ becomes even again and $\left\langle\mathcal{N}_{n p}\right\rangle$ is reduced more significantly by the increased strength of the $n n$ condenstate.

This picture augments that described in Ref. [1] and it is natural to ask how much it has to do with reality. Since the strengths of the individual pairing modes and even the total isovector pairing strength are hard to extract from the limited data available, we again turn to a large-scale shell model Monte Carlo (SMMC) calculation in the full $\mathrm{fp}$ shell to see if the physics plays out in the same way. Unfortunately in odd- $A$ and oddodd (excepting $N=Z$ ) nuclei the notorious sign-problem [4] inherent in SMMC studies with realistic interactions cannot be circumvented at low temperatures $(T \leq 0.8 \mathrm{MeV})$ by "g-extrapolation" [5]. We in large part avoid the problem, however, by using "pairing plus multipole-multipole" interaction of the kind used in Ref. [6]. In even-even nuclei this interaction has been shown to do a good job with essential features of the spectrum, including isovector pairing correlations, which have been checked against the predictions of the realistic KB3 interaction. We fix the temperature at $T=0.4 \mathrm{MeV}$, which should be sufficient to cool a nucleus to near its ground state. Although a mild sign problem still affects the nuclei considered here (the sign for ${ }^{49} \mathrm{Cr}$ is $0.35 \pm 0.01$ at $T=0.4 \mathrm{MeV}$ ) SMMC calculations can be performed without $g$-extrapolation. The only consequence of the residual sign problem is that statistical uncertainties for odd- $A$ nuclei are slightly worse than for even-even nuclei, in which there is no sign-problem at all. 


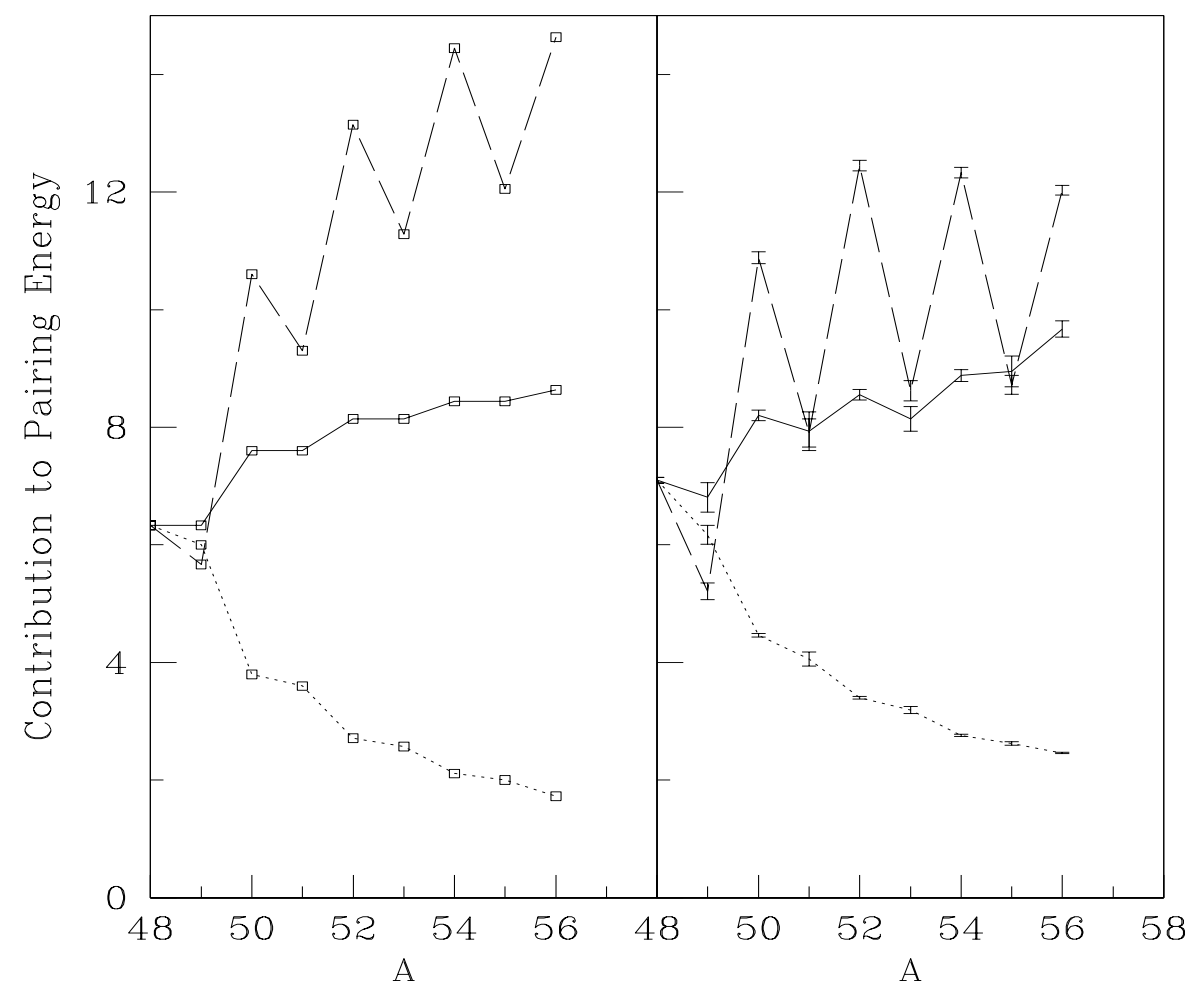

Figure 1: The quantities $\Omega\left\langle\mathcal{N}_{p p}\right\rangle$ (solid line), $\Omega\left\langle\mathcal{N}_{n n}\right\rangle$ (dashed line), and $\Omega\left\langle\mathcal{N}_{n p}\right\rangle$ (dotted line) for the $\mathrm{Cr}$ isotopes. On the left are the $\mathrm{SO}(5)$ results described in the text (with $\Omega=10$, half the number of single-particle levels in the $f p$ shell) scaled by a factor 0.5 . On the right are the results of the Shell Model Monte Carlo calculation with $G_{\text {pair }}=20 / A$ $\mathrm{MeV}$ and the quadrupole coupling constant $\chi=134 A^{-11 / 3} \mathrm{MeV} / \mathrm{fm}^{4}$.

The right-hand sides of Figure 1 contains the SMMC results for the same quantities discussed in $\mathrm{SO}(5)$, and is clearly similar to the left-hand side. The minor differences between the two panels, most apparent in $\left\langle\mathcal{N}_{n n}\right\rangle$ for large $N-Z$, are most likely due to presence of several nondegenerate levels in the $f p$ shell. We conclude, as before, that the presence of physics beyond $\mathrm{SO}(5)$ reduces the strength of each the three pairing modes by about a factor of two, but does not drastically alter the balance of power among them.

Where exactly does the factor of two come from? The shell-model contains many physical effects not included in $\mathrm{SO}(5)$, but we focus here on the role of spin-orbit splitting, which we can mock up in a two-level version of the SO(5) model. (For the formalism, applicable to seniority-zero states, see Ref. [7, 8].) Although the matrices one must diagonalize are no longer tiny, they are still small. To mimic the separation of the $f_{7 / 2}$ level from the rest of the $f p$ shell, we take our two levels to have $\Omega_{1}=4$ and $\Omega_{2}=6$ and split them by $\epsilon=10 G$, a number close to the real ratio of spin-orbit splitting to pairing-force strength. In Figure 2 we plot the numbers of the 3 kinds of pairs together with the SMMC 
predictions, now just for even-A $\mathrm{Cr}$ isotopes (i.e. for every other point in Figure 1). The splitting weakens the coherence of the pairs so that the numbers now agree quite well with the shell-model calculations without the factor of 2 scaling, except for $\left\langle\mathcal{N}_{n n}\right\rangle$ at large $N-Z$. The disagreement there clearly reflects the residual splitting between the other $f p$ levels; it doesn't appear until there are more neutrons than can be accomodated in the $f_{7 / 2}$ level. All this leads us to attribute the differences in scale between simple $\mathrm{SO}(5)$ and full shell-model calculations to spin-orbit splitting, or more generally to the nondegeneracy of the the single particle states, which can also reflect deformation.

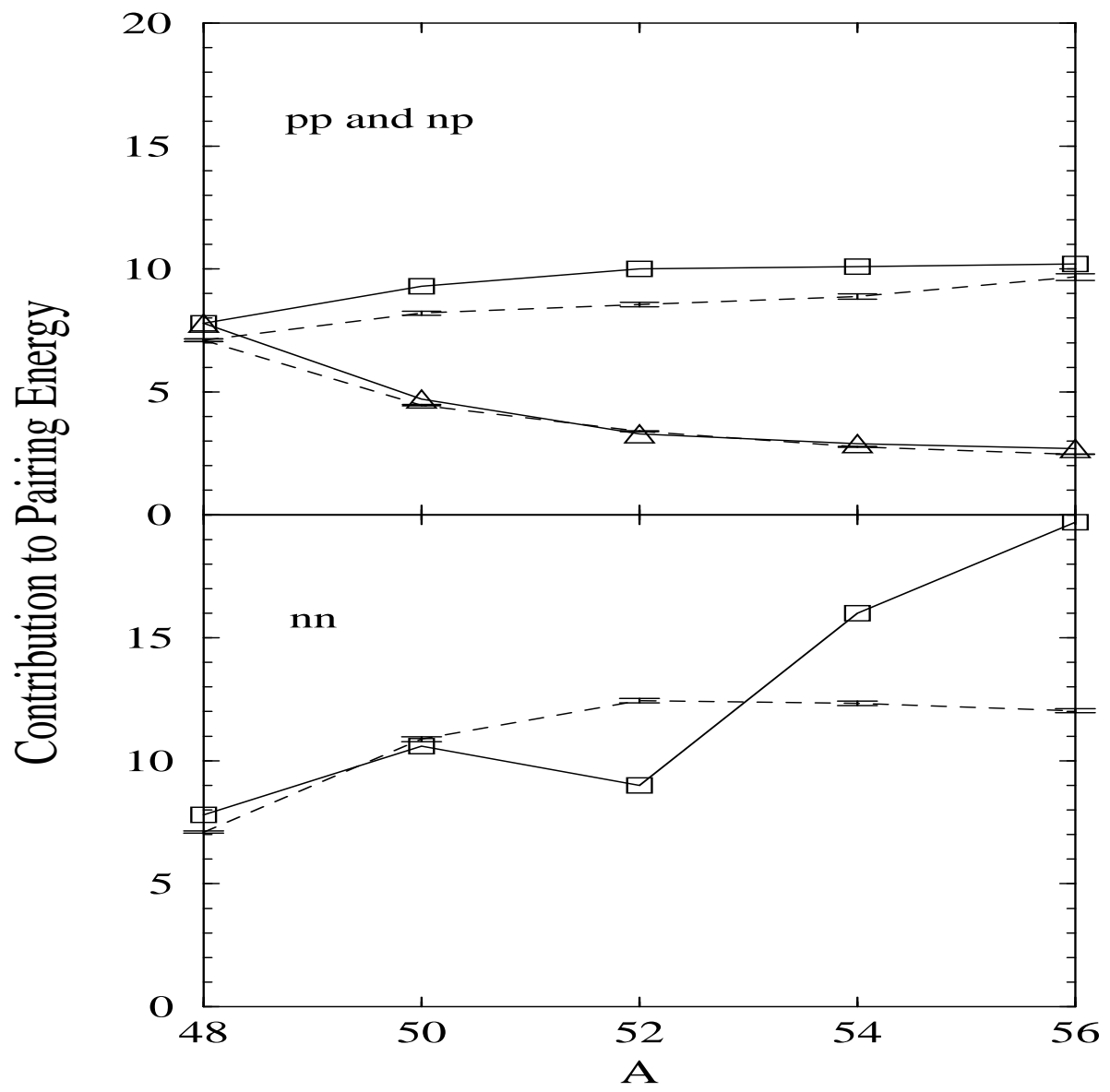

Figure 2: Comparison of the two-level model and shell model. In the upper panel are the quantities $\Omega\left\langle\mathcal{N}_{p p}\right\rangle$ and $\Omega\left\langle\mathcal{N}_{n p}\right\rangle$ and in the lower one $\Omega\left\langle\mathcal{N}_{n n}\right\rangle$. The two level model is described in the text; the results are connected by full lines. The SMMC results, with the small error bars as indicated, are connected by the dashed lines.

We turn now to the $\mathrm{Mn}$ isotopes, which have $Z=25$ and are odd-odd as well as odd-even. Figure 3 shows $\mathrm{SO}(5)$ and SMMC results for the pair numbers. The figure is split into 4 panels to separate the odd- $A$ isotopes from the even- $A$ (odd-odd) isotopes. 
The agreement between (scaled) $\mathrm{SO}(5)$ and the $\mathrm{SMMC}$ is good in the upper panels, but noticeably less so in lower panels. The problem is that the model predicts the wrong isospin for the ground states in odd-odd isotopes with $N-Z \neq 0$. The ground states in all these nuclei have $T=T_{z}$, while the model doesn't even contain such states; they appear only when a pair is broken and, if you believe the model, should lie much higher in energy.

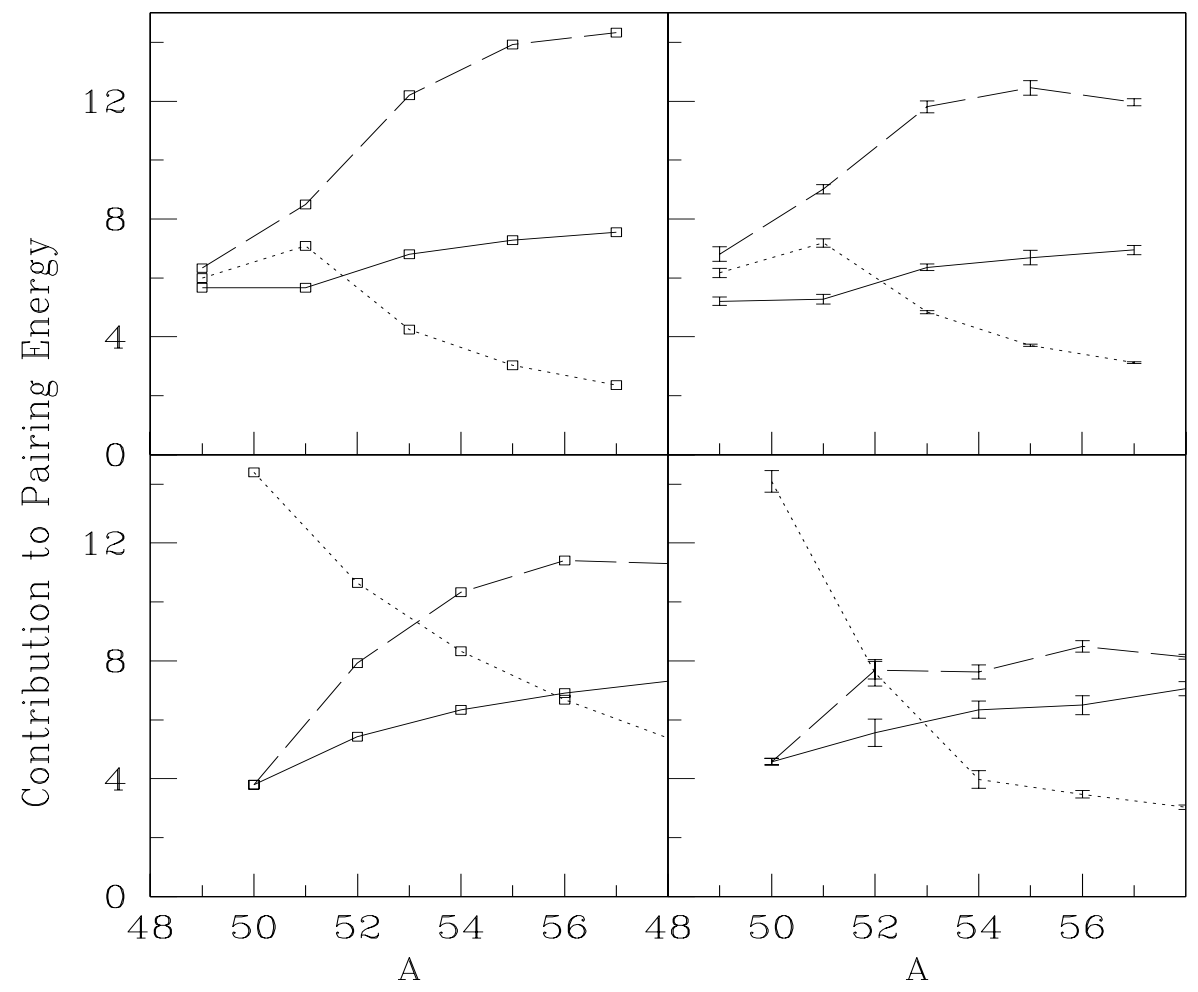

Figure 3: The quantities $\Omega\left\langle\mathcal{N}_{p p}\right\rangle$ (solid line), $\Omega\left\langle\mathcal{N}_{n n}\right\rangle$ (dashed line), and $\Omega\left\langle\mathcal{N}_{n p}\right\rangle$ (dotted line) in the odd- $A$ (top) and odd-odd (bottom) isotopes of Mn. The left side contains the $\mathrm{SO}(5)$ results, the right the SMMC results.

In the real world, however, the breaking of a pair is offset by the shell closure at $N=28$ and by the quadrupole-quadrupole force, for the following reason: When $N \geq 28(A \geq 53$ here) the lowest-lying states in the pure single-particle model have $T=T_{z}$ because the filled neutron shell prohibits the operator $\tau_{+}$from giving anything but zero. Higher $T$ states correspond to particle-hole excitations, which have too much single-particle excitation energy to be pulled all the way down when the pairing interaction is added. A similar argument in the Nilsson scheme implies that $T=T_{z}$ in the deformed nucleus ${ }^{52} \mathrm{Mn}$ as well. Our version of SO(5) includes only fully paired states even in the two-level model, and so overlooks the effects of single-particle splitting and deformation. As a result, its description of odd-odd nuclei with $N \neq Z$ is lacking. I

\footnotetext{
${ }^{1}$ Interestingly, the SMMC with our chosen strength of the pairing plus quadrupole interaction also fails
} 
We turn finally to the temperature dependence of pairing correlations in proton-rich nuclei (related work on nuclei with $N=Z$ nuclei appears in Refs. [6, 9]). As an example we have chosen ${ }^{49} \mathrm{Cr}$, an odd- $A$ nucleus with $N=Z+1$. We again use the SMMC with the Hamiltonian and model space described above to calculate pair-numbers, but now for temperatures spanning the range $T=0.4-2 \mathrm{MeV}$. Figure 4 shows the results. The blocking effect is clearly visible in the pair correlations: at low temperatures the neutron-neutron correlations are smaller than both the proton-neutron and proton-proton correlations, despite the presence of five neutrons to only four protons. As the temperature increases both the pairing strengths and the blocking effect decrease, so that for $T>1.2$ $\mathrm{MeV}$ the neutron-neutron pairing correlations are the largest and the neutron-proton the weakest, just as if there were no blocking. It appears that although both pairing correlations and Pauli blocking decrease with temperature, the latter disappear first.

\section{$49 \mathrm{Cr}$}

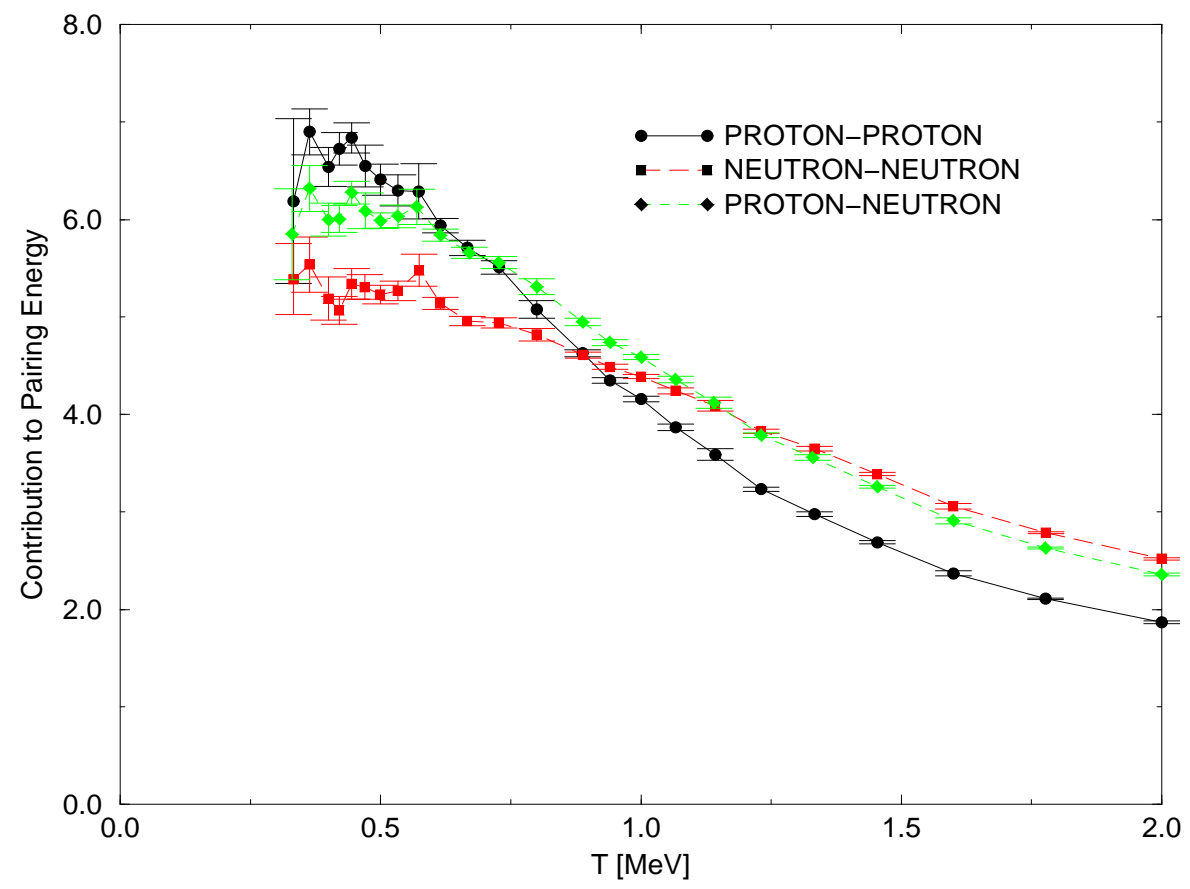

Figure 4: The quantities $\Omega\left\langle\mathcal{N}_{p p}\right\rangle$ (circles), $\Omega\left\langle\mathcal{N}_{n n}\right\rangle$ (squares), and $\Omega\left\langle\mathcal{N}_{n p}\right\rangle$ (diamonds) in the odd- $A$ nucleus ${ }^{49} \mathrm{Cr}$ as a function of temperature calculated in the SMMC.

One can imagine using functional methods to study temperature-dependence in $\mathrm{SO}(5)$ as well, provided some higher seniority states are included. We defer that exercise, however, and instead repeat in closing that although $\mathrm{SO}(5)$ predicts the wrong isospin for

to predict the correct ground-state isospin in ${ }^{52} \mathrm{Mn}$. The problem is clearly in the quadrupole-quadrupole force, which is a little too weak. Strengthening it by about $10 \%$ pulls the $T=T_{z}=1$ state below the $T=2$ state, as it is in reality. 
odd-odd nuclei with $N \neq Z$, it makes the competition among the three kinds of isovector pairs in all other isotopes very easy to understand. Most of the effects that escape the model are recovered by a simple modification - two sets of degenerate levels instead of

one - even when Pauli blocking, which affects all three modes and the competition among them, is at play.

We were supported in part by the U.S. Department of Energy under grants DE-FG0594ER40827 and DE-FG03-88ER-40397, by the U.S. National Science Foundation under grants PHY94-12818 and PHY94-20470, and by the Danish Research Council.

\section{References}

[1] J. Engel, K. Langanke, and P. Vogel, Phys. Lett. B389, 211 (1996).

[2] J. Dobes, Phys.. Lett. B413, 239 (1997).

[3] K.T. Hecht, The Vector Coherent State Method and Its Application to Problems of Higher Symmetries (Springer-Verlag, Berlin Heidelberg, 1987).

[4] S.E. Koonin, D.J. Dean and K. Langanke, Physics Reports 278, 1 (1997).

[5] Y. Alhassid, D.J. Dean, S.E. Koonin, G. Lang and W.E. Ormand, Phys. Rev. Lett. 72, 613 (1994).

[6] K. Langanke, P. Vogel and D.-C. Zheng, Nucl. Phys. A626 (1997) 735.

[7] G. G. Dussel, E. Maqueda, R. P. J. Perazzo, and J. A. Evans, Nucl. Phys. A450, 164 (1987).

[8] O. Civitarese, M. Reboiro, and P. Vogel, Phys. Rev. C56, 1840 (1997).

[9] K. Langanke, D.J. Dean, P.B. Radha and S.E. Koonin, Nucl. Phys. A613 (1997) 253. 